\title{
Clq-Mediated Repression of Human Monocytes Is Regulated by Leukocyte-Associated Ig-Like Receptor 1 (LAIR-1)
}

\author{
Myoungsun Son and Betty Diamond
}

Center for Autoimmune and Musculoskeletal Diseases, Feinstein Institute for Medical Research, Manhasset, New York, United States of America

\begin{abstract}
Systemic lupus erythematosus (SLE) is a chronic autoimmune disease characterized by abnormal function of both the innate and the adaptive immune system, leading to a loss of tolerance to self-antigens. Monocytes are a key component of the innate immune system and are efficient producers of multiple cytokines. In SLE, inappropriate activation of monocytes is thought to contribute to the loss of self-tolerance. In this study, we demonstrate that type 1 interferon (IFN) production by CpGchallenged monocytes can be suppressed by Clq through activating leukocyte-associated Ig-like receptor-1 (LAIR-1), which contains immunoreceptor tyrosine-based inhibition motifs (ITIMs). The phosphorylation of LAIR-1 and the interaction of LAIR-1 with SH2 domain-containing protein tyrosine phosphatase-1 (SHP-1) were enhanced after LAIR-1 engagement by Clq. Moreover, engagement of LAIR-1 by Clq inhibited nuclear translocation of interferon regulatory factor (IRF)-3 and IRF5 in CPGstimulated monocytes. These data suggest a model in which LAIR-1 engagement by Clq helps maintain monocyte tolerance, specifically with respect to Toll-like receptor-9-mediated monocyte activation.
\end{abstract}

Online address: http://www.molmed.org

doi: $10.2119 /$ molmed.2014.00185

\section{INTRODUCTION}

Early studies on the pathogenesis of systemic lupus erythematosus (SLE) focused on the adaptive immune system, since B and T lymphocyte abnormalities were thought to be the primary cause of autoimmunity. However, it is now increasingly recognized that components of the innate immune system also play an essential role in SLE (1-5).

Monocytes are myeloid cells that play a key role in innate immunity and are efficient producers of proinflammatory cytokines and type 1 interferons (IFNs), IFN $\alpha$ and IFN $\beta$, when stimulated by pathogen-associated molecular patterns (PAMPs) such as unmethylated bacterial DNA or damage-associated molecular patterns (DAMPs) such as apoptotic debris $(2,5,6)$. Numerous monocyte defects involving aberrant activation and dysregulation of cytokine production have been identified in SLE patients $(1,3)$. Notably, increased levels of type 1 IFN are seen in virtually all pediatric patients and a substantial percentage of adult patients. High IFN levels are a feature of some unaffected first-degree relatives as well $(7,8)$.

TLR9, expressed by B cells, macrophages, monocytes, dendritic cells (DCs) and plasmacytoid DCs (pDCs), recognizes $\mathrm{CpG}$, which mimics bacterial DNA (9-13). CpG 2216 is a prototype of the class of CpG (CPG-A), which preferentially activates myeloid cells as opposed

\begin{abstract}
Address correspondence to Betty Diamond, Center for Autoimmune and Musculoskeletal Diseases, Feinstein Institute for Medical Research, 350 Community Drive, Manhasset, NY 11030. Phone: 516-562-3830; Fax: 516-562-2953; E-mail: bdiamond@nshs.edu. Submitted September 15, 2014; Accepted for publication September 17, 2014; Epub (www.molmed.org) ahead of print September 17, 2014.
\end{abstract}

\section{The Feinstein Institute for Medical Research} Empowering Imagination. Pioneering Discovery. to B cells (14). Human monocytes exposed to CpG-A can differentiate into DCs and produce a number of cytokines including interleukin (IL)-6, IL-12, tumor necrosis factor (TNF)- $\alpha$ and type I IFN (15). When TLR9 associates with CpG motifs in the endosome, it recruits MyD88; the TLR9/MyD88 complex leads to activation of interferon regulatory factors (IRFs) $(16,17)$. IRFs including IRF3, IRF5 and IRF7 are phosphorylated and translocate into the nucleus, where they regulate transcription of type 1 IFN mRNA. IRF3 and IRF8 cooperatively regulate IFN $\beta$ production in monocytes stimulated with TLR ligands such as LPS (TLR4), Pam3csk4 (TLR2) or viral infection (18), whereas IRF3 cooperates with IRF7 to regulate IFN $\beta$ production in pDCs on TLR9 stimulation (19). Secreted type 1 IFNs bind to the IFN receptor (IFNR) acting in an autocrine manner to induce the expression of a set of secondary IFN response genes (IFN signature genes [ISGs]) such as Mx1 and OAS1 (20). Expression of these genes is tightly regulated by type 1 IFNs through the consensus IFN-stimulated response elements $(20,21)$. IRF5 also regulates tran- 
scription of the proinflammatory cytokines IL-6 and TNF $\alpha$ (22); IRF5 and nuclear factor (NF)-кB p50 coregulate IL-6 in TLR9-stimulated human plasmacytoid DCs (pDCs) (23). Genetic polymorphisms of IRF3, IRF5 and IRF7 have been associated with susceptibility to SLE $(17,24)$ and elevated levels of nuclear IRF5 have been demonstrated in monocytes of SLE patients (4).

A Src family kinase (SFK)-driven tyrosine phosphorylation pathway at the plasma membrane is upstream of and required for TLR9/MyD88 activation in endosomes (12). This result suggests that a potential CpG-sensing receptor is localized at the plasma membrane and may activate SFKs. Two SFKs, Hck and Lyn, are phosphorylated in monocytes after stimulation by $\mathrm{CpG}$ and induce actin cytoskeleton reorganization. They also activate the TLR9/MyD88 signaling cascade (12). The activation of SFKs is implemented through the catalytic activity of the kinase domain and through proteinprotein interactions of the regulatory SH2 and SH3 domains $(25,26)$. Regulation of SFKs is modulated by C-Src kinase (Csk), which phosphorylates the C-terminal tyrosine of SFK, resulting in a catalytically inactive conformation (27). Although much is understood regarding the production of IFN downstream of TLR9, the membrane proximal molecular events that suppress these pathways to prevent overproduction of cytokines have not been well described.

Leukocyte-associated Ig-like receptor-1 (LAIR-1) is an inhibitory immune receptor with immunoreceptor tyrosine-based inhibition motifs (ITIMs). It is expressed on human myeloid and lymphoid cells, including NK cells, T cells, B cells and monocytes; monocyte-derived DCs; and $\mathrm{CD} 4^{+}$hematopoietic progenitor cells (28-32). LAIR-1 engagement inhibits the differentiation of peripheral blood precursors into DCs $(33,34)$. On antibodymediated cross-linking, the tyrosines in the ITIMs of LAIR-1 become phosphorylated; phosphorylation of both ITIMs is required for full inhibition of cellular activation $(30,35)$. Phosphorylation of
LAIR-1 is inhibited by PP2, a SFK inhibitor, suggesting that the kinase responsible for LAIR-1 phosphorylation is an SFK such as Lck, Hck or Lyn (36). Both human and mouse LAIR-1 are associated with Csk $(30,35)$. Engagement of LAIR-1 recruits SH2 domain-containing protein tyrosine phosphatase-1 (SHP-1), which negatively regulates intracellular signaling (36). In NK cells, SHP-1 is associated with LAIR-1 upon stimulation by monoclonal anti-LAIR-1 antibody (31). Engagement of LAIR-1 by collagen recruits SHP-1, which negatively regulates intracellular signaling (36). SHP-1 appears to be important for negative regulation of type 1 IFN production and consequent protection against SLE-like inflammation $(24,37)$.

The complement component $\mathrm{C} 1 \mathrm{q}$ and extracellular matrix collagens are functional ligands for LAIR-1 and directly inhibit immune cell activation $(34,38,39)$. $\mathrm{C} 1 \mathrm{q}$ has been shown to regulate cytokine production and MyD88-dependent TLRmediated signaling in murine bone marrow-derived DCs (40) and to inhibit IFN $\alpha$ production by human pDCs in response to stimulation by TLR ligands in vitro (34). Moreover, C1q deficiency has been associated with abnormal production of IFN $\alpha$ by pDCs in SLE patients (41).

Here, we set out to define how $\mathrm{C} 1 \mathrm{q}$ suppresses CpG-mediated activation of monocytes. First, we demonstrate that CpG-induced production of type 1 IFN by monocytes is suppressed by C1q or anti-LAIR-1 monoclonal antibodies (mAbs) and is characterized by less nuclear translocation of IRFs. C1q-mediated suppression did not occur in cells transfected with LAIR-1 siRNA, confirming the importance of LAIR-1 to this inhibitory pathway. CpG alone augmented an interaction between LAIR-1 and the inactive form of Hck. However, when LAIR-1 was engaged by C1q or anti-LAIR-1 antibody in CpG-stimulated monocytes, the interaction between Hck and LAIR-1 was abrogated, and an interaction between SHP-1 and LAIR-1 was evident. Interestingly, we also found that
LAIR-1 mediates a ligand-independent suppression of cytokine production. These findings demonstrate that $\mathrm{C} 1 \mathrm{q}$ and LAIR-1 are dynamically involved in monocyte homeostasis.

\section{MATERIALS AND METHODS}

\section{Reagents}

Fluorochrome-conjugated and unconjugated antibodies were purchased: phycoerythrin (PE)-labeled or unlabeled mouse anti-human LAIR-1 (DX26; BD Pharmingen [BD Biosciences, San Jose, CA, USA]) for flow cytometry or stimulation; goat anti-human LAIR-1 (T-15, Santa Cruz Biotechnology, Dallas, TX, USA) for Western blotting; mouse anti-SHP-1 (D-11, Santa Cruz Biotechnology); rabbit anti-Hck (Abcam, Cambridge, MA, USA); mouse anti-phosphoSrc (Invitrogen [Thermo Fisher Scientific Inc., Waltham, MA, USA]); rabbit antiIRF3 (Cell Signaling Technology, Danvers, MA, USA); rabbit anti-IRF5 (Protein Tech, Chicago, IL, USA); mouse anti-phospho-IkB $\alpha$ (Ser32/36; Cell Signaling Technology); mouse anti-phosphoIkB $\alpha$ (Cell Signaling Technology); mouse anti-lamin A/C (Sigma-Aldrich, St. Louis, MO, USA); mouse anti- $\beta$ actin (AC-15, Sigma-Aldrich); rabbit antiGAPDH (anti-glyceraldehyde-3phosphate dehydrogenase) (Cell Signaling Technology); anti-CD11b, anti-CD14 and isotype-matched control antibodies (BD Pharmingen [BD Biosciences]); and human Fc receptor blocking solution (Biolegend, San Diego, CA, USA). Cell culture reagents included the following: Ficoll-plaque plus (GE Healthcare, London, UK); penicillin/streptomycin, RPMI 1640, L-glutamine and HEPES (all from Gibco/Invitrogen [Thermo Fisher Scientific]); heat-inactivated fetal bovine serum (FBS) (Hyclone, Logan, UT, USA); and X-VIVO serum-free media (Lonza, Basel, Switzerland). Other reagents used included: $\mathrm{C} 1 \mathrm{q}$ from Complement Technology (Tyler, TX, USA); CpG 2216 (Invitrogen [Thermo Fisher Scientific]); 1× RIPA cell lysis buffer (Invitrogen [Thermo Fisher Scientific]); 
protease inhibitor cocktail (Pierce, Waltham, MA, USA); phosphatase inhibitors (Pierce); formaldehyde, Triton X-100 and NP-40 (Sigma-Aldrich); Tween-20 (Fisher Scientific [Thermo Fisher Scientific]); and phosphatebuffered saline (PBS) and distilled water (Gibco/Invitrogen [Thermo Fisher Scientific]). Proteins and culture reagents were endotoxin-tested ( $<0.1$ Endotoxin Units $[\mathrm{EU}] / \mathrm{mL}$ ) either by the manufacturer or by using a limulus amebocyte lysate assay kit performed per the manufacturer's instructions (Charles River Endosafe, Kingston, NY, USA).

\section{Human Monocyte Isolation, Culture and Stimulation}

Human peripheral blood mononuclear cells (PBMCs), obtained according to institutional guidelines of the Feinstein Institute for Medical Research, were isolated from the blood of healthy donors by density centrifugation (New York Blood Center, New York, NY, USA). Monocytes were negatively enriched by using a human monocyte enrichment kit (Stemcell Technologies, Vancouver, BC, Canada). Purity of monocytes ( $90 \%$ $\left.\mathrm{CD}_{11 b^{+}} \mathrm{CD}_{14}{ }^{+}\right)$was assayed by flow cytometry. Purified monocytes $\left(1 \times 10^{6}\right.$ cells $/ \mathrm{mL}$ ) were immediately stimulated for indicated times until $30 \mathrm{~min}$ with CpG 2216 (5 $\mu \mathrm{mol} / \mathrm{L})$, C1q $(25 \mu \mathrm{g} / \mathrm{mL})$ or anti-LAIR-1 antibody $(10 \mu \mathrm{g} / \mathrm{mL})$ in $X$-VIVO serum-free medium and harvested at the indicated time points. For cytokine assay, cells were cultured in U-bottom 96-well plates (Nunc, Waltham, MA, USA) containing complete media (RPMI-1640 containing $2 \mathrm{mmol} / \mathrm{L}$ L-glutamine, $10 \mathrm{mmol} / \mathrm{L}$ HEPES, $50 \mathrm{IU} / \mathrm{mL}$ penicillin, $50 \mathrm{mg} / \mathrm{mL}$ streptomycin and 10\% FBS).

\section{Quantitative Real-Time Polymerase Chain Reaction}

Total RNA was extracted with an RNeasy kit (Qiagen, Hilden, Germany) and subjected to reverse transcription with an iScript cDNA synthesis kit (BioRad, Hercules, CA, USA). cDNA was analyzed by quantitative polymerase chain reaction (qPCR) by using a LightCycler 480 master mix with TaqMan probes (Applied Biosystems [Thermo Fisher Scientific]) against human IFN $\alpha 4$ (Hs01681284), IFNß1 (Hs01077958), MX1 (Hs00182073), OAS1 (Hs00242942), IL-6 (Hs00985639), TNF $\alpha$ (Hs00174128) and Polr2A (Hs00172187). Data were normalized to Polr2a; relative induction was calculated by $\Delta \Delta \mathrm{Ct}$.

\section{Cytokine Assay}

Cytokine analysis for IL-6 and TNF $\alpha$ was performed using a Human ProInflammatory 7-Plex Ultra-Sensitive Kit (Meso Scale Discovery [MSD], Rockville, MD). MSD plates were analyzed on the MS2400 imager (MSD). Assay was performed according to the manufacturer's instructions. All standards and samples were measured in duplicate.

\section{Isolation of Nuclear Fractions}

The NE-PER Nuclear and Cytoplasmic Extraction Kit (Thermo Scientific

[Thermo Fisher Scientific]) was used for nuclear extraction following the manufacturer's protocol. The efficiency of nuclear fractions was determined by immunoblotting for lamin.

\section{Confocal Microscopy}

Unstimulated or stimulated monocytes were washed three times with ice-cold PBS, fixed with $4 \%(\mathrm{v} / \mathrm{v})$ paraformaldehyde and permeablized with $0.2 \%$ Triton X-100. Cells were seeded $\left(2 \times 10^{5}\right.$ cells $\left./ \mathrm{mL}\right)$ on slides by using cytospin and blocked with $2 \%$ goat serum and $2 \%$ bovine serum albumin (Invitrogen [Thermo Fisher Scientific]). Cells were incubated overnight at $4^{\circ} \mathrm{C}$ with anti-IRF3 antibody (1:100) and washed and incubated with Alexa Fluor 488-labeled goat anti-mouse antibody (1:200; Invitrogen [Thermo Fisher Scientific]). Nuclei were stained with propidium iodide $(0.5 \mu \mathrm{g} / \mathrm{mL}$; Sigma-Aldrich). Cells were washed and mounted, and confocal images were captured by FluoView 300 (Olympus, Center Valley, PA, USA) and analyzed by using Axio vision 4.8 software (Zeiss, Jena, Germany).

\section{Transfection and Flow Cytometry}

In RNA interference assays, monocytes were transfected by using an AmaxaNucleofector kit (Lonza). Transfection efficiency was over $40 \%$. siRNAs for LAIR-1 or control siRNA were from Qiagen. The target sequence of human LAIR-1 is CAGCATCCAGAAGGTTCGTTA. The efficiency of knockdown was determined by flow cytometry (FACS verse; BD Biosciences) with PE-labeled anti-human LAIR-1 antibody. Data were analyzed by using FlowJo software (Tree Star; see http:/ /www.flowjo.com/).

\section{Immunoprecipitation and Western Blot Analysis}

Unstimulated or CpG-stimulated monocytes $\left(2-5 \times 10^{6}\right.$ cells $\left./ \mathrm{mL}\right)$ were washed in ice-cold PBS and lysed in $1 \times$ RIPA buffer containing complete protease inhibitor mixture (Roche, Basel, Switzerland) and phosphatase inhibitor (Pierce). For immunoprecipitation, cell lysates were then diluted 10-fold with lysis buffer and anti-LAIR-1 antibody before being incubated with precleared protein G-dynabeads (Life Technologies [Thermo Fisher Scientific]). Immunoblotting of bound proteins was performed with the indicated antibodies.

\section{Phospho-Immunoreceptor Array}

To determine tyrosine phosphorylated LAIR-1, cell lysates from monocytes $(7 \times$ $10^{6}$ ) stimulated without $\mathrm{CpG}$ or with CpG only, CpG plus C1q or CpG plus anti-LAIR-1 antibody were incubated with human phosphoimmunoreceptor array membranes (Proteome Profiler Array; R\&D Systems, Minneapolis, MN, USA) according to the manufacturer's protocol. Phosphorylation levels of individual analytes were determined by average pixel density of duplicate spots; values were obtained after subtraction of background and were normalized to positive control spots.

\section{Statistical Analysis}

Statistical analyses were performed with Prism 5.0 (GraphPad, La Jolla, CA, USA). $P$ values were calculated by using 
A
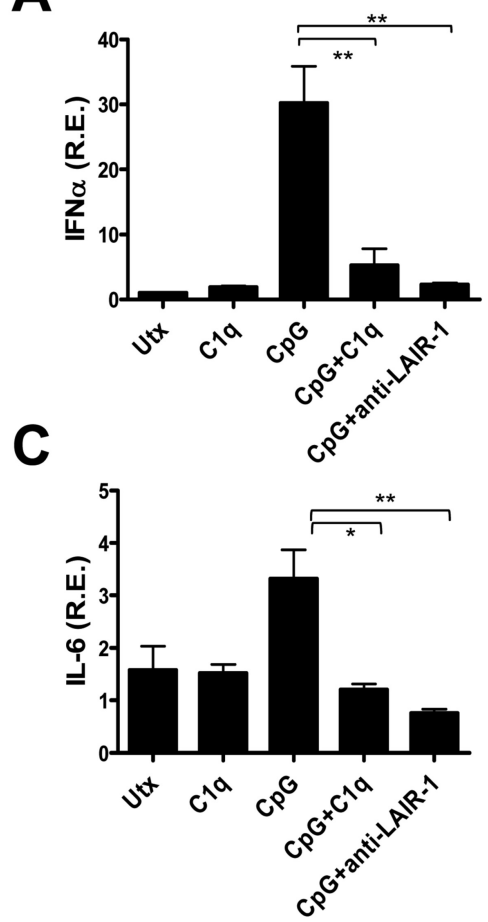

B
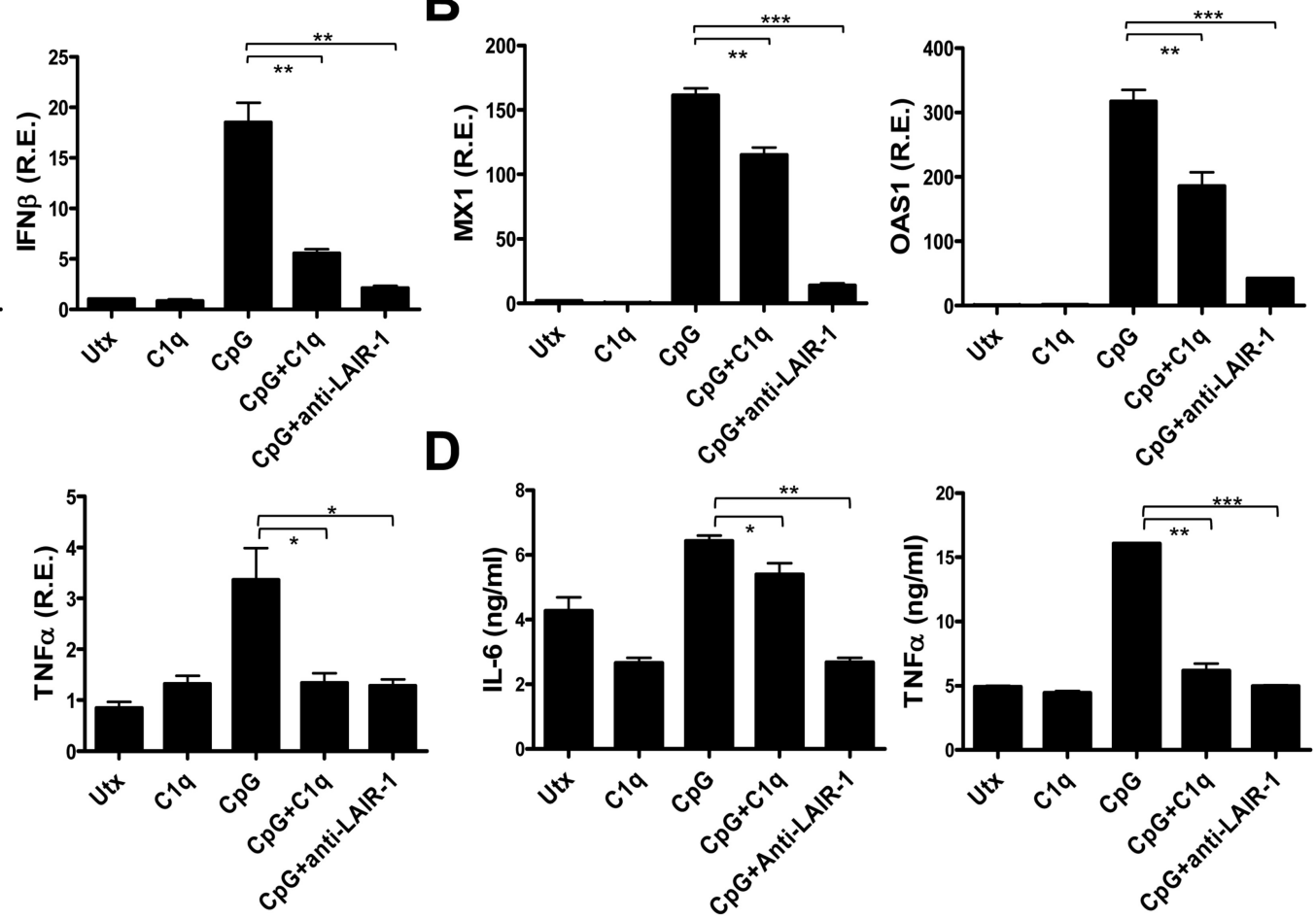

Figure 1. LAIR-1 engagement by Clq or anti-LAIR-1 suppresses CpG-triggered production of cytokines in human monocytes. Purified human monocytes from healthy donors were unstimulated or stimulated with CpGODN 2216 (CpG), Clq with CpG or anti-LAIR-1 antibody with CPG for $4 \mathrm{~h}$. Transcripts were determined by qRT-PCR. Clq or anti-LAIR-1 antibody inhibited CpG-mediated IFN $\alpha$ and IFN $\beta$ induction (A), IFN signature genes MXI and OASl (B) and CpG-mediated IL-6 and TNF $\alpha$ induction (C). (D) After $24 \mathrm{~h}$ of culture, supernatants were collected, and the production of IL-6 and TNF $\alpha$ was measured by MSD assay. Utx, untreated. R.E., relative expression. Results represent the means \pm standard error of the mean (SEM); the data were obtained from three independent experiments. ${ }^{*} P<0.05,{ }^{* *} P<$ $0.01,{ }^{* *} P<0.001$.

a two-tailed unpaired Student $t$ test or two-way ANOVA. $P$ values of $<0.05$ were considered significant: ${ }^{*} p<0.05,{ }^{* *} p<$ 0.01 and ${ }^{* * *} p \leq 0.001$ (Student $t$ test).

\section{RESULTS}

\section{Clq and LAIR- 1 Modulate the Expression of Multiple Genes in Human Monocytes Stimulated with CpG}

We have previously shown that the addition of C1q to human pDCs inhibited IFN $\alpha$ production after $\mathrm{CpG}$ stimulation in a dose-dependent fashion. In addition, this inhibition was reversed by soluble LAIR-2 (34), suggesting that the inhibitory effects of $\mathrm{C} 1 \mathrm{q}$ required that $\mathrm{C} 1 \mathrm{q}$ bind to LAIR-1. Besides pDCs, human monocytes also highly express LAIR-1 and are efficient producers of type 1 IFN and NF-кB-dependent cytokines after TLR9 ligation. We examined a potential role of the LAIR-1 signaling cascade activated by $\mathrm{C} 1 \mathrm{q}$ or anti-LAIR-1 mAb in regulating the expression of type 1 IFNs (IFN $\alpha$ and IFN $\beta$ ) and the ISGs MX1 and OAS1 in human monocytes. We stimulated monocytes in serum-free medium lacking C1q, with CpG ODN 2216, which activates TLR9 (14). Significantly, IFN and ISGs were induced by $4 \mathrm{~h}$ after $\mathrm{CpG}$ stimulation, but not after C1q stimulation alone. Stimulation with CpG together with $\mathrm{C} 1 \mathrm{q}$ led to diminished transcription of IFN and downstream ISGs (Figures 1A, B). The proinflammatory cytokines IL-6 and TNF $\alpha$ were also induced by $\mathrm{CpG}$ exposure and inhibited at the mRNA and protein level in the presence of C1q (Figures 1C, D). Anti-LAIR-1 antibody added to CpG-stimulated cells, but not control mouse IgG, also inhibited induction of IFN, ISGs and proinflammatory cytokines, suggesting that LAIR-1 engagement might be the mechanism for C1q-induced suppression.

To confirm the importance of LAIR-1, we used LAIR-1 siRNA to decrease LAIR-1 expression (Figure 2C). While cells transfected with control siRNA responded to $\mathrm{CpG}$ with increased expression of IFN $\alpha$ and showed an inhibition of $\mathrm{CpG}$ activation in the presence of $\mathrm{C} 1 \mathrm{q}$ or anti-LAIR-1 antibody, cells transfected with LAIR-1 siRNA exhibited a higher basal level of IFN $\alpha$ transcription and less inhibition by $\mathrm{C} 1 \mathrm{q}$ or anti-LAIR-1 antibody. The fact that basal IFN $\alpha$ transcription was not increased in cells transfected with control siRNA suggests that the increase in IFN $\alpha$ transcription present in LAIR-1 siRNA-transfected mono- 


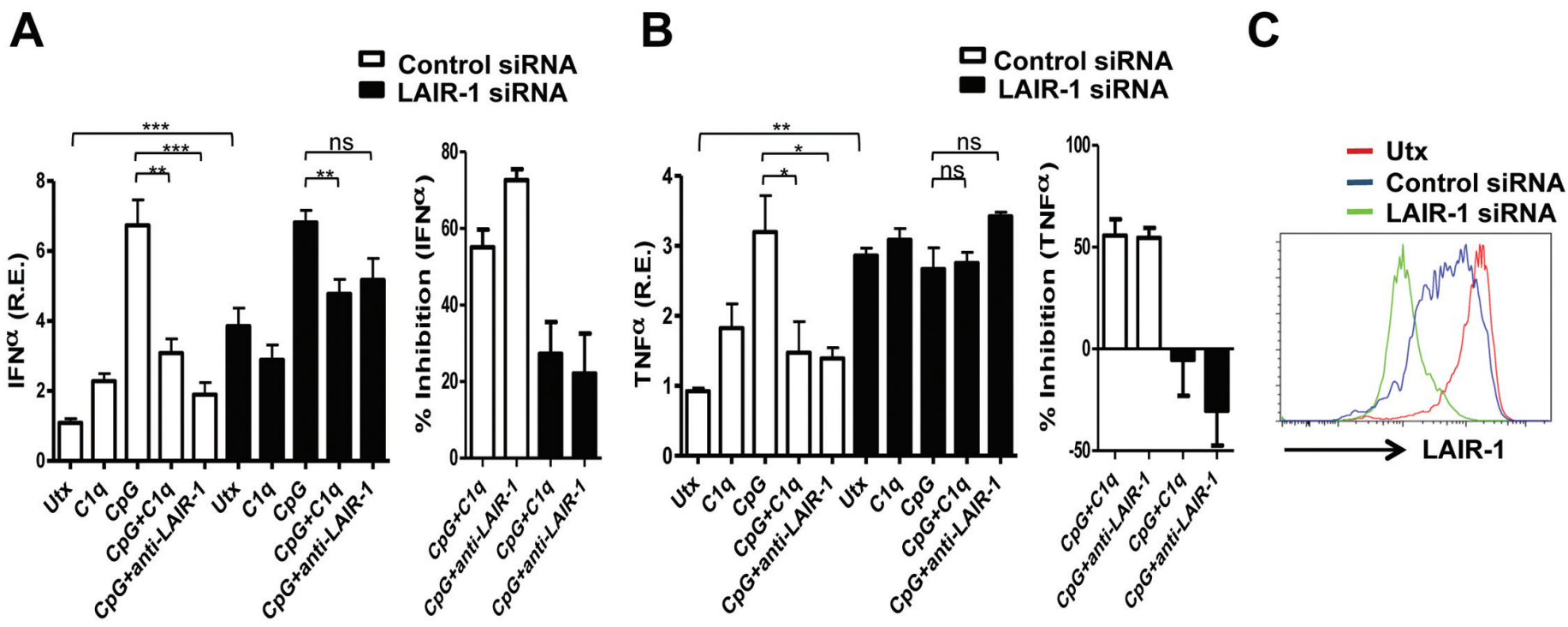

Figure 2. Clq-meditated suppression of IFN $\alpha$ and TNF $\alpha$ production is reversed by LAIR-1 siRNA. (A, B) Human monocytes were transfected with control siRNA or LAIR-1 siRNA. After 24 h, cells were stimulated with CpG, C1q, CpG plus Clq or CpG and anti-LAIR-1 antibody for 4 h. Transcripts of IFN $\alpha$ and TNF $\alpha$ were determined by qRT-PCR. Results represent mean \pm SEM; the data were obtained from three independent experiments. ${ }^{*} P<0.05,{ }^{* *} P<0.01,{ }^{* * *} P<0.0001$, ns, not significant. Percent inhibition was calculated as based on qRT-PCR. (C) The efficiency of siRNA was determined at $24 \mathrm{~h}$ by flow cytometry with PE-labeled anti-LAIR-1 antibody.

cytes was not due to engagement of TLRs by siRNA, but rather that LAIR-1 has a constitutive C1q-independent inhibitory function in monocytes. TNF $\alpha$ transcription was increased by $\mathrm{CpG}$ stimulation in monocytes transfected with control siRNA and was inhibited by C1q or anti-LAIR-1 antibody (Figure 2B). Basal TNF $\alpha$ transcription was increased in monocytes transfected with LAIR-1 siRNA, and there was no discernable increase on exposure to $\mathrm{CpG}$; thus, there was no inhibition by C1q or anti-LAIR-1 antibody (Figure 2B). The data indicate that C1q and LAIR-1 have an important regulatory function in controlling $\mathrm{cy}$ tokine production in response to $\mathrm{CpG}$ challenge in monocytes.

\section{Clq and LAIR-1 Inhibit the Nuclear Translocation of IRFs Mediated by CpG Stimulation}

Because the transcription of type 1 IFNs and ISGs is strongly regulated by IRFs, we studied the activation of these molecules after CpG stimulation of human monocytes. We isolated nuclear fractions from human monocytes that had been stimulated with $\mathrm{CpG}$, with or without C1q or anti-LAIR-1 antibody. CpG caused an increased nuclear localization of IRF3 and IRF5, which was not seen in the presence of $\mathrm{C} 1 \mathrm{q}$ or anti-LAIR-1 antibody (Figure 3A). We used confocal microscopy to confirm our observations on the subcellular localization of IRF3. In unstimulated monocytes or monocytes treated with $\mathrm{C} 1 \mathrm{q}$ alone, IRF3 localized to the cytoplasm. When CpG was added to monocytes, almost all detectable IRF3 was translocated to the nucleus (Figure 3B). The effect of CpG on IRF3 nuclear translocation was suppressed by coincubation with C1q or anti-LAIR-1 antibody. Approximately $75 \%$ of cells retained cytoplasmic IRF3 in the presence of $\mathrm{C} 1 \mathrm{q}$ (Figure 3B). As shown in Figure 3C, phosphorylation of $\mathrm{IkB} \alpha$, which releases the cytoplasmic retention of NF- $\mathrm{KB}$, was enhanced by $\mathrm{CpG}$ stimulation and reduced after $\mathrm{CpG}$ stimulation in the presence of $\mathrm{C1q}$ or anti-LAIR-1 antibody. These data suggest that C1q and LAIR-1 signaling negatively regulates the nuclear translocation of the key transcription factors for cytokine gene activation. Nuclear IRFs bind to responsive elements in the promoters in type 1 IFN genes. Furthermore, IRFs cooperate with NF-кB to induce proinflammatory cytokines. Thus, C1q mediates suppression of monocytes to produce cytokines through these two important pathways.

\section{LAIR-1 Sequesters the Inactive Hck during CpG-Mediated Activation While Clq Leads to SHP- 1 Engagement with LAIR-1}

We next asked how LAIR-1 negatively regulates CpG-mediated activation of monocytes. To determine which proteins might be associated with LAIR-1, we performed coimmunoprecipitation studies. We found that Csk is associated with LAIR-1 in unstimulated monocytes, and this association is present also in CpG-stimulated monocytes (Figure 4A). Because Csk is known to phosphorylate the inhibitory tyrosine of SFKs, we tested for SFK phosphorylation at this site. We found that inactive SFK was associated with LAIR-1 in the resting state and following $\mathrm{CpG}$ exposure (Figure 4A). We observed Hck to be the major binding partner for LAIR-1 in both unstimulated and CpG-stimulated 

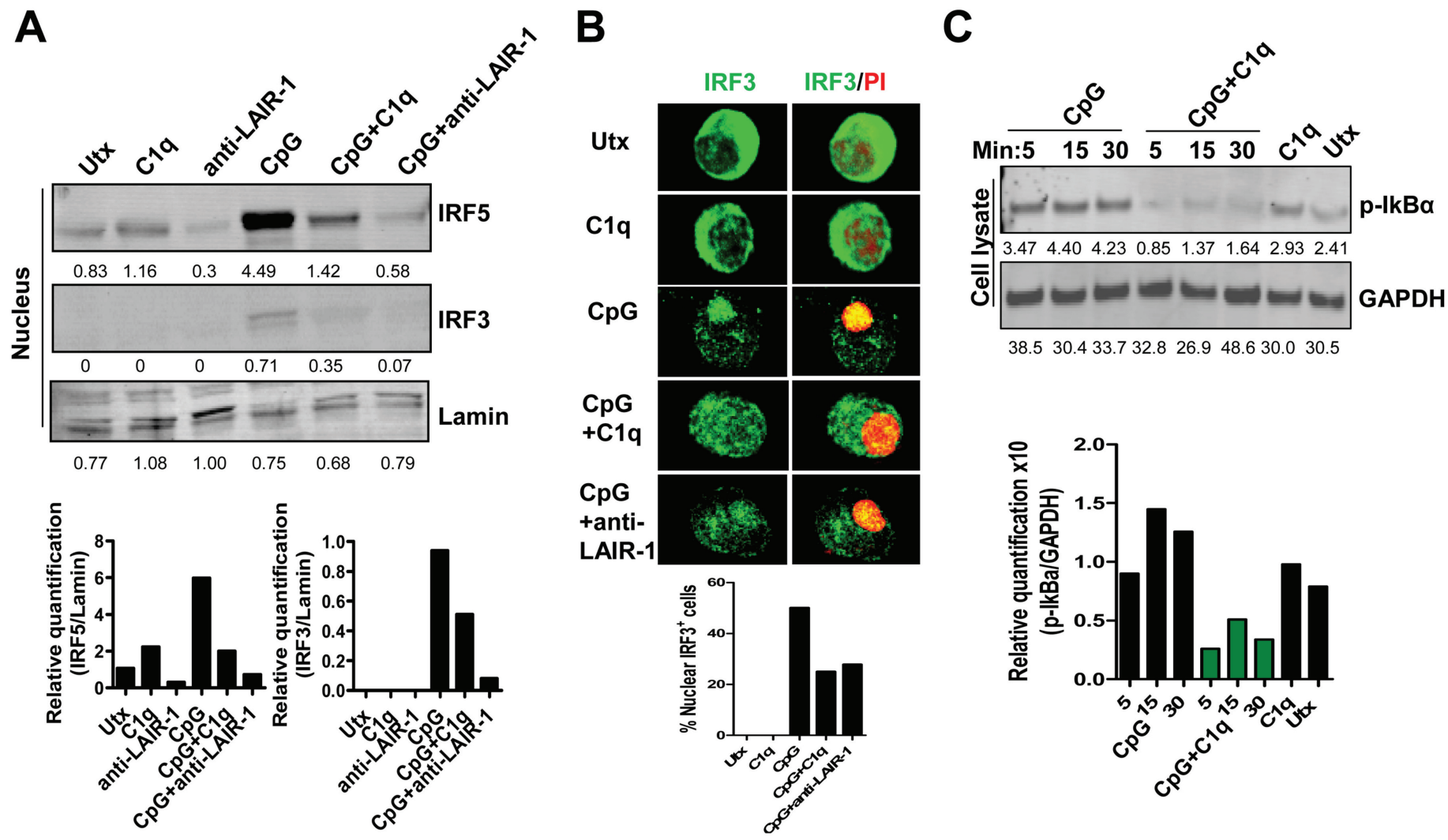

Figure 3. LAIR-1 engagement inhibits CpG-mediated nuclear translocation of IRFs and the activation of NF-kB. Human monocytes were stimulated with CPG, Clq or anti-LAIR-1 antibody for $3 \mathrm{~h}$. Nuclear fractions were analyzed by Western blot for IRF5, IRF3 and lamin for a marker for the nuclear fraction. Clq and anti-LAIR-1 antibody inhibit CpG-mediated nuclear translocation of IRF5 and IRF3 (A). Data are representative of three independent experiments. (B) Stimulated cells were stained for IRF3 and propodium iodine (PI) as a nuclear marker. The percentage of IRF3 nuclear translocation was assessed in more than 200 cells in three independent experiments, and representative images are shown. All images were viewed at 120x magnification by using a FluoView 300 confocal microscope (Olympus). (C) Purified monocytes were stimulated with $\mathrm{CPG}$ or $\mathrm{Clq}$ together with $\mathrm{CpG}$ for the indicated times, and cell lysates were assayed for phosphorylated-lkB $\alpha$ by Western blotting. Phosphorylated IkBa was normalized to GAPDH. Experiments were repeated three times, and representative data are shown.

monocytes. When LAIR-1 was engaged by C1q or anti-LAIR-1 antibody in CpGstimulated monocytes, the interaction between Hck and LAIR-1 was abrogated, and an interaction between SHP-1 and LAIR-1 was enhanced (Figure 4A).

To explore the dynamic interactions of these proteins, we performed the same coimmunoprecipitation study at different time points after CpG stimulation in the absence or presence of $\mathrm{C} 1 \mathrm{q}(5,15$ and 30 $\mathrm{min})$. The results of this analysis suggested that Csk constitutively binds to LAIR-1. Hck binds to LAIR-1 and is inactivated, presumably by Csk, after CpG stimulation. However, on engagement by C1q, LAIR-1 changes binding partners and associates with SHP-1 (Figure 4B).
Next we studied whether CpG stimulation or LAIR-1 engagement alters the phosphorylation of LAIR-1. We previously reported that $\mathrm{C} 1 \mathrm{q}, \mathrm{C} 1 \mathrm{q}$ collagen tail or anti-LAIR-1 antibody each induce phosphorylation of LAIR-1 (34). Here, monocytes were stimulated with $\mathrm{CpG}$, CpG plus C1q or CpG plus anti-LAIR-1 antibody. There was detectable phosphorylation of LAIR-1 even in unstimulated monocytes, perhaps accounting for the constitutive inhibition mediated by LAIR-1. CpG challenge modestly increased LAIR-1 phosphorylation, whereas LAIR-1 engagement with C1q or anti-LAIR-1 antibody strongly induced LAIR-1 phosphorylation (Figure 5). Presumably, Hck is responsible for the phos- phorylation of LAIR-1 that occurs after C1q binding.

\section{DISCUSSION}

In this study, we demonstrate a suppressive mechanism of $\mathrm{C} 1 \mathrm{q}$ in human monocytes. Monocytes have been increasingly recognized to play a role in both the initiation and propagation of SLE given their functions in phagocytosis and cytokine production $(1,5,6,42)$. In particular, in the pristane-induced model of murine lupus, monocytes play a key pathogenic role by acting as a major producer of type I IFNs (5). Human monocytes express TLR9 and respond to CpG $(13,43)$. We observed that C1q or anti-LAIR-1 antibody inhibited CpG- 
A
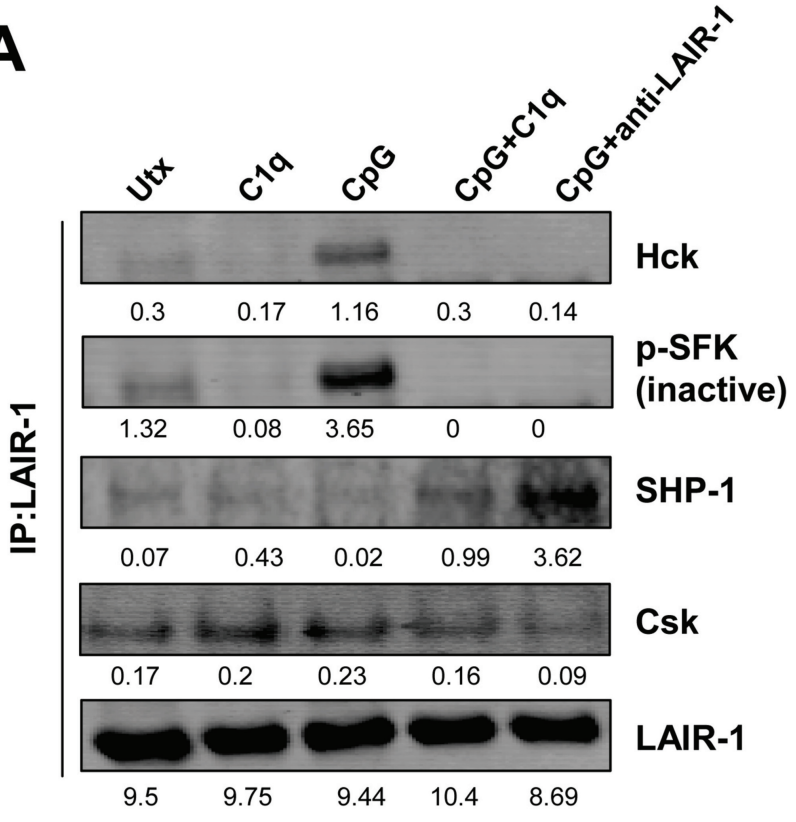

B

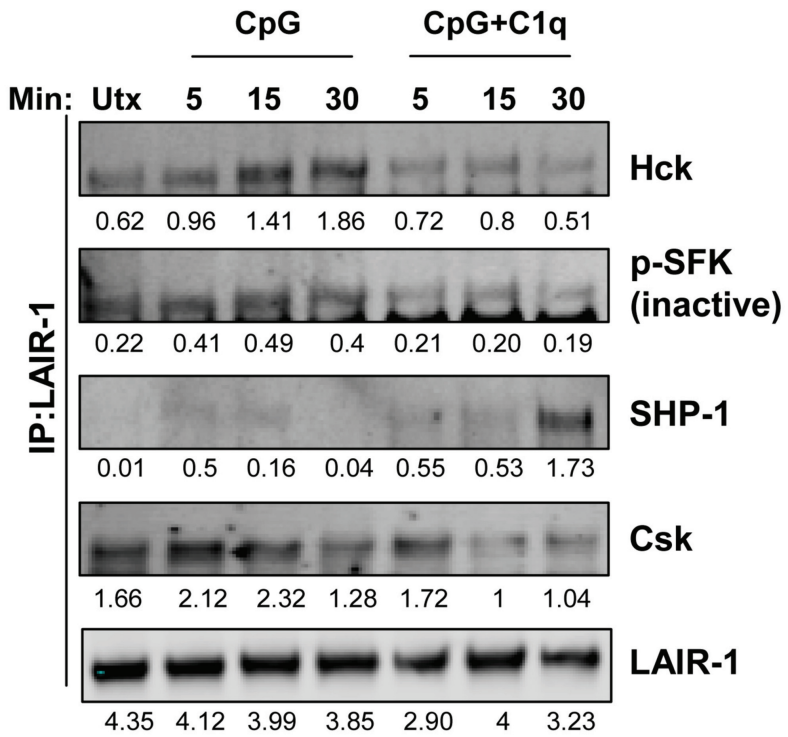

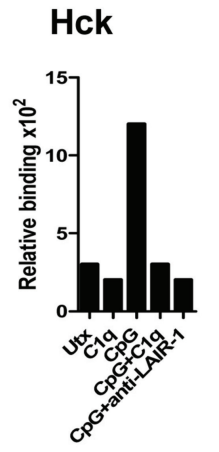
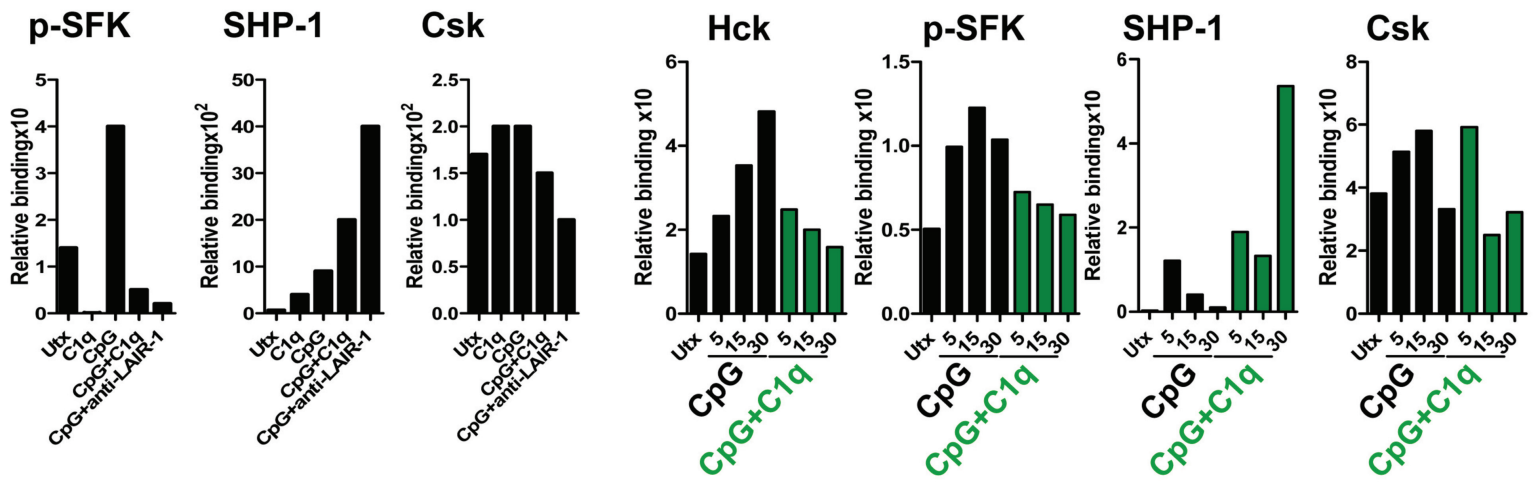

Figure 4. LAIR-1 engagement induces an interaction between LAIR-1 and SHP-1, but CPG stimulation induces an interaction of Hck with LAIR-1. (A) Human monocytes were stimulated with CpG, Clq, CpG plus Clq or CpG plus anti-LAIR-1 antibody for 30 min. Cell lysates were immunoprecipitated with anti-LAIR-1 antibody, and LAIR-1 binding proteins were analyzed by Western blot. Data are representative of three independent experiments. (B) Cells were stimulated for the indicated time, and the same experiment as in (A) was performed. Numbers in blots represent density, and relative bindings were calculated by the ratio for the immune-precipitated LAIR-1. Experiments were repeated three times, and representative data are shown.

mediated nuclear translocation of IRF3 and IRF5 and phosphorylation of IkB $\alpha$. The fact that the suppression of CpGmediated activation of monocytes by C1q was reversed by LAIR-1-specific siRNA demonstrated that the suppressive effects of C1q are LAIR-1 dependent. Transfection of monocytes with LAIR-1 siRNA led to higher baseline levels of cytokine transcription, demonstrating a role for LAIR-1 in monocyte homeostasis. Moreover, CpG did not increase cytokine expression in monocytes lacking LAIR-1 expression, suggesting that CpG stimulation blocks the constitutive inhibitory function of LAIR-1. Our findings demonstrate a contribution of C1q deficiency to SLE that is independent of the role of $\mathrm{C} 1 \mathrm{q}$ in clearance of apoptotic debris.

To identify the proteins that interact with LAIR-1 after TLR9 activation with or without LAIR-1 engagement, we performed immunoprecipitation studies by using anti-LAIR-1 antibody. Csk constitutively associates with LAIR-1. We ob- served an increased association of inactive Hck with LAIR-1 upon CpG treatment; presumably Csk phosphorylates the C-terminal tyrosine of Hck to produce the inactive form that we observed. We propose that $\mathrm{CpG}$ activates Csk leading to phosphorylation of Y522 and inactivation of Hck on LAIR-1. Binding sites have been identified on LAIR-1 for Hck (Y251) (VTYAQL) and Csk (Y281) (ITYAAV). We also observed increased phosphorylation of the LAIR-1 ITIM motifs after CpG exposure, but phosphory- 


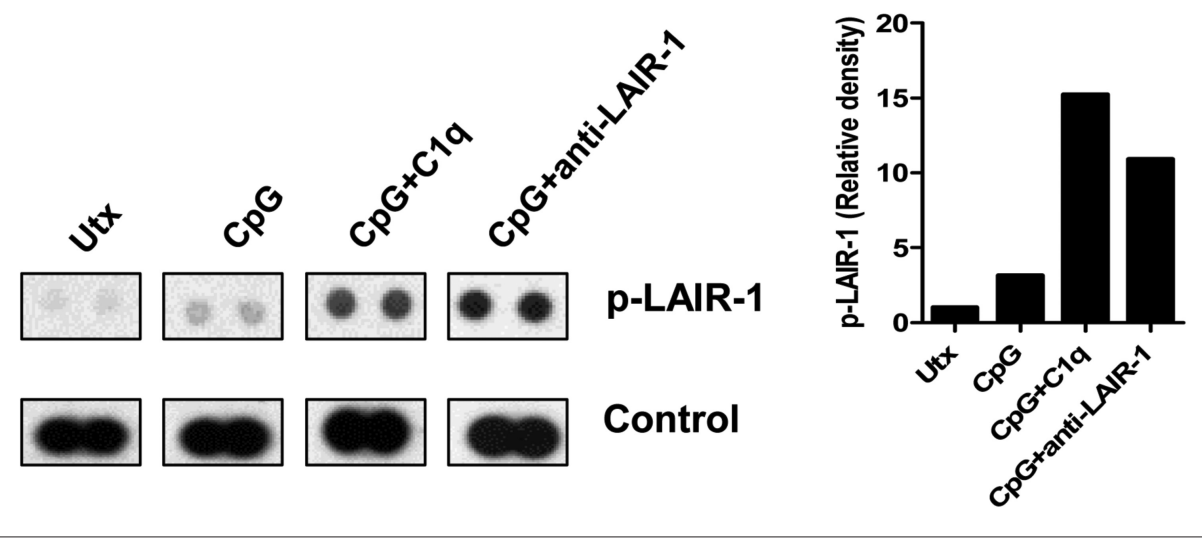

Figure 5. LAIR-1 engagement induces the tyrosine phosphorylation. The state of LAIR-1 phosphorylation was measured by a proteome profile array-human phospho-immunoreceptor assay. Whole cell extracts from untreated (Utx), CpG, CpG plus Clq or CpG plus anti-LAIR-1 antibody were analyzed. The experiment was repeated three times, and representative data are shown. Relative quantification for the phosphorylation of LAIR-1 was normalized to control spots.

lation was even greater in the presence of C1q or anti-LAIR-1 antibody. We propose that C1q engages LAIR-1, causing Hck to be activated, perhaps auto-catalytically, and leading to the phosphorylation of the ITIM motifs of LAIR-1. This step permits binding to SHP-1. SHP-1 binds to both ITIM motifs when they are phosphorylated. In this way, C1q and LAIR-1 mediate an inhibitory pathway modulating TLR9 activation (Figure 6). LAIR-1 mediates partial inhibition in a ligand-independent fashion. There may be some SHP-1 associated with LAIR-1 in the resting state. This association is not detected in our coimmunoprecipitation studies and is eliminated when $\mathrm{CpG}$ is present. After LAIR-1 engagement by C1q, phosphorylated LAIR-1 recruits SHP-1 and induces the phosphatase activity of SHP-1. SHP-1 dephosphorylates the activating tyrosine residues of SFKs, as well as various other intracellular tyrosine phosphorylation substrates.

IFNs are key mediators of host defense to viral and bacterial infection. They are also thought to be involved in the pathogenesis of SLE $(17,20,44,45)$. Patients treated with IFN for hepatitis C infection develop anti-nuclear antibodies or overt SLE (46). Serum levels of IFN correlate with disease activity in SLE. We demonstrate that C1q and LAIR-1 signaling suppresses the activation of IRFs, and this mechanism regulates the transcription of multiple cytokines upon CpG stimulation in human monocytes.

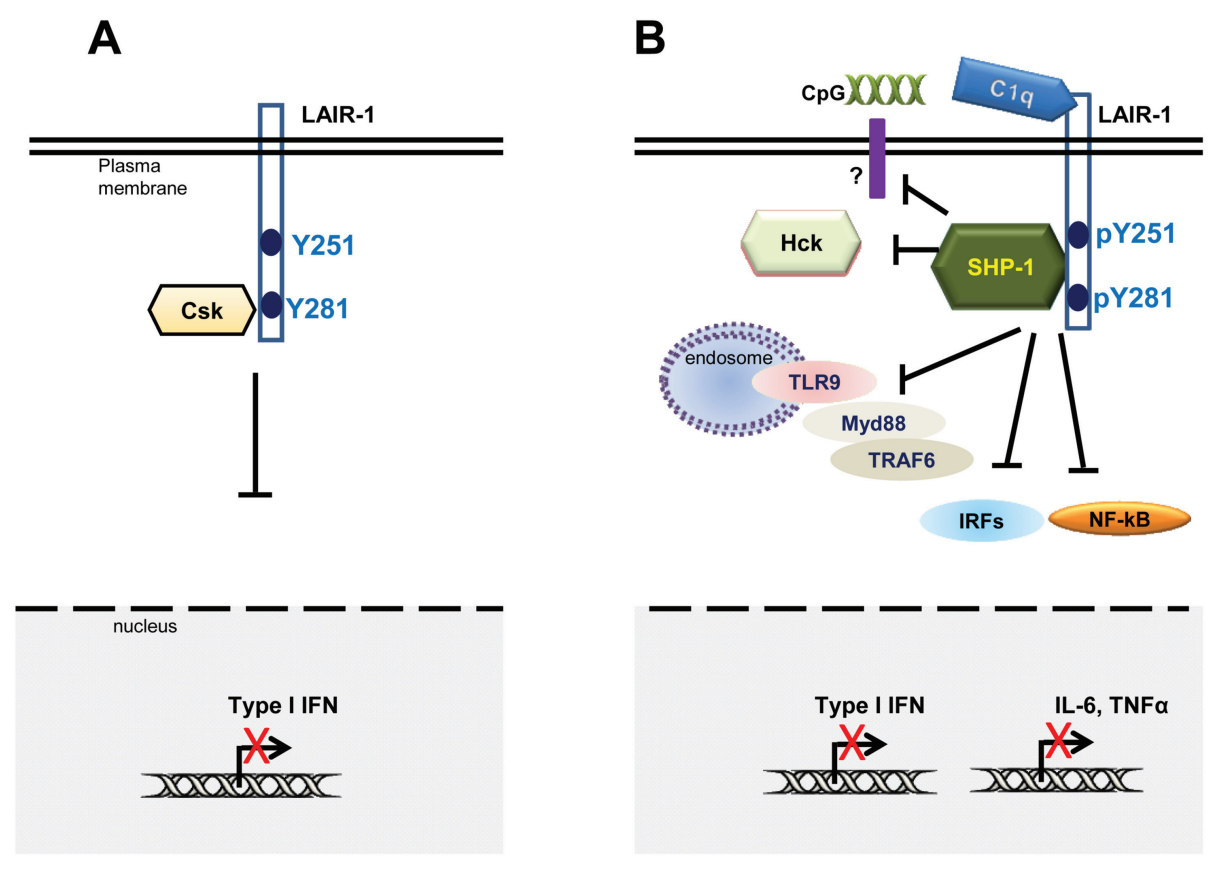

Figure 6. Model demonstrating how LAIR-1 contributes to immune tolerance. $(A)$ In the resting state, Csk constitutively associates with LAIR-1. (B) When LAIR-1 is engaged in the presence of Clq, Hck phosphorylates the ITIMs on LAIR-1. Phosphorylated LAIR-1 recruits SHP-1 and induces the phosphatase activity of SHP-1. SHP-1 mediates the inhibition of monocytes by dephosphorylating the activating tyrosine residues of various intracellular tyrosine-phosphorylation substrates and by inhibiting nuclear translocation of IRFs or NF-kB resulting in cytokine production. 
tail, which engages LAIR-1, inhibits TLR signaling. A recent report showed that collagenous domains of C-type lectin surfactant protein-D (SP-D) bind to LAIR-1 and regulate FcoR-mediated reactive oxygen production in neutrophils (48). These results suggest that LAIR-1 engagement by collagen-like domains may be a therapeutic strategy for controlling inflammation in SLE and inflammatory conditions.

\section{ACKNOWLEDGMENTS}

This work was supported by National Institutes of Health Grant R01AR-049126 to B Diamond and an Arthritis Foundation Fellowship to M Son. We would like to thank Sylvia Jones for expert secretarial assistance and the flow cytometry and imaging cores of the Feinstein Institute for Medical Research and Barbara Sherry, Frances Santiago-Schwarz, Sun-Jung Kim and Yong-Rui Zou for review of the manuscript. We also thank Frances SantiagoSchwarz for initiating our studies of LAIR-1.

\section{DISCLOSURE}

The authors declare that they have no competing interests as defined by Molecular Medicine, or other interests that might be perceived to influence the results and discussion reported in this paper.

\section{REFERENCES}

1. Katsiari CG, Liossis SN, Sfikakis PP. (2010) The pathophysiologic role of monocytes and macrophages in systemic lupus erythematosus: a reappraisal. Semin. Arthritis Rheum. 39:491-503.

2. Lee PY, et al. (2008) A novel type I IFN-producing cell subset in murine lupus. J. Immunol. 180:5101-8.

3. Li Y, Lee PY, Reeves WH. (2010) Monocyte and macrophage abnormalities in systemic lupus erythematosus. Arch. Immunol. Ther. Exp. (Warsz). 58:355-64.

4. Stone RC, et al. (2012) Interferon regulatory factor 5 activation in monocytes of systemic lupus erythematosus patients is triggered by circulating autoantigens independent of type I interferons. Arthritis Rheum. 64:788-98.

5. Yang L, Feng D, Bi X, Stone RC, Barnes BJ. (2012) Monocytes from Irf5-/- mice have an intrinsic defect in their response to pristane-induced lupus. J. Immunol. 189:3741-50.

6. Hansmann L, Groeger S, von Wulffen W, Bein G,
Hackstein H. (2008) Human monocytes represent a competitive source of interferon-alpha in peripheral blood. Clin. Immunol. 127:252-64.

7. Kanakoudi-Tsakalidou F, et al. (2014) Simultaneous changes in serum HMGB1 and IFN-alpha levels and in LAIR-1 expression on plasmatoid dendritic cells of patients with juvenile SLE: new therapeutic options? Lupus. 23:305-12.

8. Niewold TB, Hua J, Lehman TJ, Harley JB, Crow MK. (2007) High serum IFN-alpha activity is a heritable risk factor for systemic lupus erythematosus. Genes Immun. 8:492-502.

9. Ahmad-Nejad P, et al. (2002) Bacterial CpG-DNA and lipopolysaccharides activate Toll-like receptors at distinct cellular compartments. Eur. J. Immunol. 32:1958-68.

10. Bauer S, et al. (2001) Human TLR9 confers responsiveness to bacterial DNA via species-specific CpG motif recognition. Proc. Natl. Acad. Sci. U. S. A. 98:9237-42.

11. Hemmi H, et al. (2000) A Toll-like receptor recognizes bacterial DNA. Nature. 408:740-5.

12. Sanjuan MA, et al. (2006) CpG-induced tyrosine phosphorylation occurs via a TLR9-independent mechanism and is required for cytokine secretion. J. Cell Biol. 172:1057-68.

13. Saxena M, Busca A, Pandey S, Kryworuchko M, Kumar A. (2011) CpG protects human monocytic cells against HIV-Vpr-induced apoptosis by cellular inhibitor of apoptosis- 2 through the calcium-activated JNK pathway in a TLR9independent manner. J. Immunol. 187:5865-78.

14. Kerkmann M, et al. (2003) Activation with CpGA and CPG-B oligonucleotides reveals two distinct regulatory pathways of type I IFN synthesis in human plasmacytoid dendritic cells. J. Immunol. 170:4465-74.

15. Shirota H, Klinman DM. (2012) Effect of CpG ODN on monocytic myeloid derived suppressor cells. Oncoimmunology. 1:780-2.

16. Gilliet M, Cao W, Liu YJ. (2008) Plasmacytoid dendritic cells: sensing nucleic acids in viral infection and autoimmune diseases. Nat. Rev. Immunol. 8:594-606.

17. Salloum R, Niewold TB. (2011) Interferon regulatory factors in human lupus pathogenesis. Transl. Res. 157:326-31.

18. Li P, et al. (2011) IRF8 and IRF3 cooperatively regulate rapid interferon-beta induction in human blood monocytes. Blood. 117:2847-54.

19. Yang H, Ma G, Lin CH, Orr M, Wathelet MG. (2004) Mechanism for transcriptional synergy between interferon regulatory factor (IRF)-3 and IRF-7 in activation of the interferon-beta gene promoter. Eur. J. Biochem. 271:3693-703.

20. Delgado-Vega AM, Alarcon-Riquelme ME, Kozyrev SV. (2010) Genetic associations in type I interferon related pathways with autoimmunity. Arthritis Res. Ther. 12 Suppl 1:S2.

21. Honda K, Taniguchi T. (2006) IRFs: master regulators of signalling by Toll-like receptors and cytosolic pattern-recognition receptors. Nat. Rev. Immunol. 6:644-58.
22. Takaoka A, et al. (2005) Integral role of IRF-5 in the gene induction programme activated by Tolllike receptors. Nature. 434:243-9.

23. Steinhagen F, et al. (2013) IRF-5 and NF-kappaB p50 co-regulate IFN-beta and IL-6 expression in TLR9-stimulated human plasmacytoid dendritic cells. Eur. J. Immunol. 43:1896-906.

24. Akahoshi M, et al. (2008) Promoter polymorphisms in the IRF3 gene confer protection against systemic lupus erythematosus. Lupus 17:568-74.

25. Schwartzberg PL. (1998) The many faces of Src: multiple functions of a prototypical tyrosine kinase. Oncogene. 17:1463-8.

26. Rhee I, Veillette A. (2012) Protein tyrosine phosphatases in lymphocyte activation and autoimmunity. Nat. Immunol. 13:439-47.

27. Okada M, Nada S, Yamanashi Y, Yamamoto T, Nakagawa H. (1991) CSK: a protein-tyrosine kinase involved in regulation of src family kinases. J. Biol. Chem. 266:24249-52.

28. Bonaccorsi I, et al. (2010) The immune inhibitory receptor LAIR-1 is highly expressed by plasmacytoid dendritic cells and acts complementary with NKp44 to control IFNalpha production. PLoS One. 5:e15080.

29. van der Vuurst de Vries AR, Clevers H, Logtenberg T, Meyaard L. (1999) Leukocyte-associated immunoglobulin-like receptor-1 (LAIR-1) is differentially expressed during human B cell differentiation and inhibits B cell receptor-mediated signaling. Eur. J. Immunol. 29:3160-7.

30. Meyaard L. (2008) The inhibitory collagen receptor LAIR-1 (CD305). J. Leukoc. Biol. 83:799-803.

31. Meyaard L, et al. (1997) LAIR-1, a novel inhibitory receptor expressed on human mononuclear leukocytes. Immunity. 7:283-90.

32. Tang $X$, et al. (2012) Leukocyte-associated Ig-like receptor-1-deficient mice have an altered immune cell phenotype. J. Immunol. 188:548-58.

33. Poggi A, Tomasello E, Ferrero E, Zocchi MR, Moretta L. (1998) p40/LAIR-1 regulates the differentiation of peripheral blood precursors to dendritic cells induced by granulocyte-monocyte colonystimulating factor. Eur. J. Immunol. 28:2086-91.

34. Son M, Santiago-Schwarz F, Al-Abed Y, Diamond B. (2012) C1q limits dendritic cell differentiation and activation by engaging LAIR-1. Proc. Natl. Acad. Sci. U. S. A. 109:E3160-7.

35. Verbrugge A, Ruiter Td T, Clevers H, Meyaard L. (2003) Differential contribution of the immunoreceptor tyrosine-based inhibitory motifs of human leukocyte-associated Ig-like receptor-1 to inhibitory function and phosphatase recruitment. Int. Immunol. 15:1349-58.

36. Xu M, Zhao R, Zhao ZJ. (2000) Identification and characterization of leukocyte-associated Ig-like receptor-1 as a major anchor protein of tyrosine phosphatase SHP-1 in hematopoietic cells. J. Biol. Chem. 275:17440-6.

37. Khaled AR, Butfiloski EJ, Sobel ES, Schiffenbauer J. (1998) Functional consequences of the SHP-1 defect in motheaten viable mice: role of NFkappa B. Cell. Immunol. 185:49-58. 
CI q MEDIATES MONOCYTE INHIBITION VIA LAIR-I

38. Elkon KB, Santer DM. (2012) Complement, interferon and lupus. Curr. Opin. Immunol. 24:665-70.

39. Lood C, et al. (2009) C1q inhibits immune complexinduced interferon-alpha production in plasmacytoid dendritic cells: a novel link between C1q deficiency and systemic lupus erythematosus pathogenesis. Arthritis Rheum. 60:3081-90.

40. Yamada M, et al. (2004) Complement C1q regulates LPS-induced cytokine production in bone marrow-derived dendritic cells. Eur. J. Immunol. 34:221-30.

41. Santer DM, et al. (2010) C1q deficiency leads to the defective suppression of IFN-alpha in response to nucleoprotein containing immune complexes. J. Immunol. 185:4738-49.

42. Byrne JC, et al. (2012) Genetics of SLE: functional relevance for monocytes/macrophages in disease. Clin. Dev. Immunol. 2012:582352.

43. Izaguirre A, et al. (2003) Comparative analysis of IRF and IFN-alpha expression in human plasmacytoid and monocyte-derived dendritic cells. J. Leukoc. Biol. 74:1125-38.

44. Hooks JJ, et al. (1979) Immune interferon in the circulation of patients with autoimmune disease. N. Engl. J. Med. 301:5-8.

45. Banchereau J, Pascual V. (2006) Type I interferon in systemic lupus erythematosus and other autoimmune diseases. Immunity. 25:383-92.

46. Omagari K, et al. (2003) Anti-extractable nuclear antigens (ENA) antibodies in patients with chronic hepatitis $C$ before and after treatment with interferon. Autoimmunity. 36:269-73.

47. Ma CY, et al. (2012) Elevated plasma level of HMGB1 is associated with disease activity and combined alterations with IFN-alpha and TNFalpha in systemic lupus erythematosus. Rheumatol. Int. 32:395-402.

48. Olde Nordkamp MJ, et al. (2014) Leukocyte-associated Ig-like receptor-1 is a novel inhibitory receptor for surfactant protein D. J. Leukoc. Biol. 96:105-11 